\title{
Laughter as Strategies in Business Communication Between Thai and Burmese Professionals
}

\author{
Tabtip Kanchanapoomi \\ Ph.D. Graduate, Department of Language Studies, School of Liberal Arts, \\ King Mongkut's University of Technology Thonburi, Bangkok, Thailand \\ tkanchanapoomi@gmail.com \\ Wannapa Trakulkasemsuk \\ Associate Professor, Department of Language Studies, School of Liberal Arts, \\ King Mongkut's University of Technology Thonburi, Bangkok, Thailand \\ wannapa.tra@kmutt.ac.th
}

\begin{abstract}
Laughter is not just an element in human communication that signifies happiness and enjoyment, it can be used as a communication strategy to lubricate successful interaction including business communication. Nonetheless, not many studies have paid attention to laughter in business communication. Therefore, this paper sheds light on how Thai and Burmese participants used laughter in a restaurant and in a business meeting in Yangon, Myanmar. Audio data was collected together with various pieces of ethnographic data, for example, participant observations reported from extensive field notes, semi-structured interviews and audio recordings. The analysis was based on the classification of laughter adopted from Hayakawa (2003), and Murata and Hori (2007). The findings reveal that laughter is deployed as a communication strategy with different purposes such as to make fun of work, to ease tension and to threaten other interlocutors and unveil those factors which stimulate the laughter in informal and formal settings.
\end{abstract}

\section{Keywords}

laughter - international business professionals - Thai and Burmese business people - informal and formal settings - ethnographic method 


\section{Introduction}

Global mobility does not only involve economic globalization but it also entails other aspects such as business interactions, business etiquette, cultural knowledge exchange, effective communication etc.

The issue of multinational workplace communication attracted the field researcher's attention in 2014 when working for a family business in Thailand which has a joint-venture in Yangon, Myanmar. Since the field researcher had been working in the international business section with Burmese business people at the construction company in Thailand, it was observed that, when Thai and Burmese employees interacted, they appeared to have particular verbal (e.g. response: "yes") and non-verbal behaviors (e.g. smiles, laughter, nodding). Through the participant observation, laughter was identified as a striking feature which recurred in the data collected in informal and formal business meetings.

Hence, the purpose of this study is to examine the role of laughter and the factors that drove laughter to occur during an international business communication between 2 Thai and 3 Burmese professionals involved in the glass and aluminium construction field in informal (a brunch meeting at a restaurant) and formal (a business meeting at an office) settings. This group of research participants and two contexts were selected because it was interesting to study how similar or different a group of business professionals from the same region with partially shared cultural-based communication were, how laughter was included in their business dealings and how laughter was interpreted. In addition, not many studies have explored the cultural and behavioral perspectives of laughter, particularly the use of laughter between Thai and Burmese business people. Laughter is considered to be an element of communication (Bachorowski and Owren 2001) and is an embedded cultural element, hence it is possible that the laughter can convey a message and communicate specific emotions and intentions to other interlocutors (Gervais and Wilson 2005).

\section{Laughter in Business Communication}

Laughter is non-verbal behavior which frequently occurs in our daily lives and to some extent has a cultural influence on building and maintaining human relationships. Laughter has been perceived and defined by a number of scholars. These include Brain (2000) who describes laughter as a physiological response to humor which consists of a set of gestures and the production of 
a sound. With a combination of gestures and a sound, laughter supports and modifies what is said in words and conveys meanings and feelings about the speaker's emotional state to others (Ruch and Ekman 2001). Further Szameitat et al. $(2009,409)$ reflects that "Laughter, thus, is an acoustical nonverbal vocalization, which carries information about the sender's emotional state that can be decoded by the listener" (2009, 402). Not only laughter but also some physical reactions (i.e., a smile) are often provoked after something that the participants perceive as humorous (Brock 2016; Lehmann-Willenbrock and Allen 2014). According to Bonin, Campbell, and Vogel (2014, 15), laughter is possibly "a response to what has preceded in conversation or in the external context of the conversation in which it appears. Laughter may also signal what is to follow in conversation". In essence, as Murata and Hori (2007) suggest laughter is a useful component of discourse which affects human relationships and Provine $(2013,9)$ later coined the term "group bonding" to describe this condition. Laughter can happen for many reasons. For example, laughter can simply occur because others are laughing (Oveis, Spectre, Smith, Liu, and Keltner 2016); laughter can be a stand-alone utterance (Ginzburg, Breitholtz, Cooper, Hough, and Tian 2015) and it is widely believed that laughter occurs when one feels amused.

However, it is possible that laughter might not have to always be related with what is considered "humorous." According to Provine's study (2013), less than $20 \%$ of laughter incidents responded to what interlocutors perceive as funny and laughter occurred in natural breaks in conversations, functioning as a period to punctuate the speech. Therefore, his discovery suggests that laughter is a form of communication, not only a product of humor. In addition to Provine's findings, Ritter, Brùck, Jacob, Wildgruber, and Kreifelts (2015), mentioned that in humans, laughter has evolved into different types, for example joyful and taunting laughter which perform different social functions and social segregation. Thus, laughter is not only a reaction but possibly performs other functions when people are conversing.

However, not many studies in the field of business discourse emphasize the importance of laughter or other types of non-verbal communication but highlight vocabulary and grammatical knowledge as indispensable for meetings, especially international business meetings. Handford and Matous (2015) and Rogerson- Revell (2007) suggested that a lack of vocabulary and grammatical knowledge are the main cause of misunderstandings in international business settings. Forgas (1985) believed that words need more cognitive effort and more time to be prepared and decoded compared to other types of communication and that is maybe the reasons why non-verbal communication receives 
less attention when consciously observing and interpreting. However, Murata and Hori (2007) have noted that misunderstandings in intercultural communications usually occur when speakers use their own communication styles and listeners interpret them using their own norms which might not be the same as the speakers. This can lead to misperception. To explain, laughter can be used as a communication strategy in order to express the feelings of one interlocutor to another. However, when the backgrounds to the use of laughter of each interlocutor are different, it is perfectly possible that they might interpret "laughter" in dissimilar ways, which can lead to conflict and misunderstanding and affect personal and professional relationships.

Since laughter can happen for various reasons, it has become a topic of interest for scholars and has been investigated (Clift 2016; Crooke, Gorman, Myers, and Duran 2013; Glenn 2010; Lehmann-Willenbrock and Allen 2014; Lyttle 2007; Marakaki, Merlino, Mondada, and Oloff 2010; Murata 2014; Ojha and Holmes 2010; Rogerson-Revell 2007; Vöge 2008). These studies have shed light not only on the form and function of the use of laughter, but also the interpretation of laughter such as an explicit reaction to humor (Godkewitsch 1976; Meyer 200o). A feeling of embarrassment, an expression of agreement, or even laughter without clear purpose are also considered to be "a response to humor in interaction" (Vöge 2008, 49). Moreover, Hayakawa (2003) stated that apart from humor, laughter can be used as a communication strategy in contexts such as the Japanese classroom and Japanese business discourse. Her findings reveal interesting types of laughter used in these two contexts, for example, "balancing laughter for easing tension" and "laughter as a cover-up" (Hayakawa 2003, 330).

Laughter, according to Hayakawa (2003), can serve as a tool to maintain a smooth relationship between interlocutors in different contexts. This perspective has also been confirmed by Murata and Hori (2007), who discovered that laughter helps create and retain human relationships and they also described some cultural differences in the use of laughter. To illustrate, they pinpointed that the use of laughter between American students and Japanese students was different. The American students laughed only when they perceived something as "funny." On the other hand, the Japanese students laughed when they found the conversation humorous and they also laughed "without obvious reason" which the researchers categorized as "laughter following unlaughable utterance." The American students did not understand and finally decided to disregard their laughter. This demonstrates that different groups can interpret laughter differently within the same discourse if they are from different cultural backgrounds. 


\section{$3 \quad$ Methodology}

\subsection{Context of Study}

As previously mentioned in the introduction, the context of this research drew the researchers' attention after the field researcher had the opportunity to expand a Thai company's operations in the neighboring country, Myanmar, in 2013.The data of this current research was collected from two places as follows.

\subsubsection{Meeting}

The meeting took place in Yangon, Myanmar, and the meeting context was selected because it was a place where the participants' ideas were disseminated and organizational knowledge and culture were also constructed. With regard to the meeting element of this study, it was considered semi-formal since they held the discussions in an open-plan office. The research participants found this convenient because they were able easily to invite others associated with ongoing topics to join the discussion which included work and non-workrelated issues. This is in accord with earlier research where the open plan layout provided flexibility, reduced unused space and fostered a collaborative environment (Andrews 2016).

\subsubsection{Restaurant}

This, too, was in Yangon. The restaurant was also considered an interesting place to collect data as it was a source where the research participants not only met to have meals but also made small talk and discussed work. The restaurant where the field researcher and the research participants went was categorized as a fast-casual restaurant which positioned itself between a fast-food restaurant and a casual dining restaurant. It was popular amongst office workers who could have a decent meal in their breaks. Due to the fact that a restaurant setting is not formal, the topics of discussion varied - depending on the participants and situations at that time.

\subsection{Data Collection}

All procedures and data collection contributing to this work comply with the ethical standard of King Mongkut's University of Technology Thonburi (кмUTт); the owner of the company and the research participants had granted permission before the project commenced. The data used for this study was collected based on ethnographic methods. In other words, the use of audio recording, participant observation, field notes, semi-structured interviews and photographing methods was employed in order to collect the spoken data and other information for this study. The researcher decided to 
employ audio recording as it was considered "not intrusive" while they were talking and discussing work. Meanwhile the participant observation assisted the field researcher in observing interactions in the meeting and restaurant settings. The detailed field notes emerged after the field researcher revised the rough ideas on scratch notes compiled during the field work to recall what had been experienced. Further, semi-structured interviews were conducted after the audio data was transcribed and analyzed to confirm the transcription, to assist in some inaudible parts, and to elaborate their speech or their emotions at a particular moment. The field researcher also used a mobile phone to take some photos as additional assistance to recall small details such as the seating positions, surrounding atmosphere, actions and experiences. These tools and techniques capture ideas and provided insight into the research participants' attitudes towards the use of laughter by Thai and Burmese business professionals.

\subsection{Research Participants}

The research participants were Thai and Burmese professionals working in construction organizations and involved in Thai and Burmese business meetings in four different settings, at the airport, in a vehicle, at the restaurant and in the meeting. They used English as the medium of communication. The total number of participants who participated in this current study was 5 people - a Thai engineer, a Thai project coordinator, a Burmese project manager, a Burmese project coordinator and a Burmese foreman. During the conversation at the restaurant, there were three participants who joined the brunch; Th1, Th2 and M1. The following is brief information on the individual research participants.

They were interviewed to collect information in terms of educational and professional background. Moreover, they were also asked to evaluate their English-speaking skills. The names used in the transcription are pseudonyms (i.e. Th1, Th2, M1, M2). "Th" means Thailand and 'M' is Myanmar. Those who are mentioned during the discussion but were not present in the meeting, including the company names, are written under pseudonyms and are put into quotation marks for example "son," "yen," "mao," "temtem" etc.

\subsubsection{Thai Participants}

Th1: Engineer, 35 years old, He spent two years in the US studying for a diploma in business studies and a master's degree at a famous public university in Thailand. He had worked for the company for almost five years as a factory engineer. 
Th2: Project Coordinator, 34 years old. She obtained her bachelor's degree from a well-known public university in Thailand. She could speak Thai, English and Japanese. She had been working for this company for almost two years. Her responsibilities were to correspond with the Burmese parties.

\subsubsection{Burmese Participants}

M1: Project Manager, 62 years old. He finished his studies at high school in Myanmar. He had lived in Singapore and also worked in Thailand for a few years in the construction field. He was able to speak Burmese, English and Thai. He was an experienced project manager.

M2: Project Coordinator, 37 years old. He obtained his bachelor's degree in Myanmar. He had worked as a project coordinator for three years. He could speak three languages: Burmese, English and Japanese.

M3: Foreman, 30 years old. He finished his studies in Myanmar. He had worked for the company for almost two years. He could speak Burmese and English.

The topics of conversation in both settings included small talk and work issues. In the meeting, a Thai engineer led the talk with another Thai coordinator as his assistant. From participant observation, the Thai engineer could convey basic meaning on unfamiliar topics or prompt questions with a limited use of vocabulary. Meanwhile, the Thai project coordinator had the capacity to deal with unfamiliar topics, such as technical terms used in the field and she could generally guess the meaning from its context. Both Thai participants were in the meeting at all times. As for the Burmese participants, only the project manager was present throughout the meeting. During the discussion, Mi had a wider range of vocabulary with which to discuss topics at length. When there seemed to be communication problems, he was able to use different communication strategies such as giving examples, explaining and asking questions etc. The other two Burmese business people were present only when the topics related to them. One was a project coordinator and whilst he could communicate reasonably well in English, one of his problems was a lack of confidence whenever he spoke which was shown in his body language (e.g. eye-contact) and his tone of voice. The other was a foreman who could speak Burmese and a little bit of English. Even though he was able to communicate with a Thai engineer, he sometimes needed the Burmese project manager to assist him with translations from Burmese to English.

\subsection{Data Preparation}

When the data from the meeting and the restaurant was collected, it was further transcribed following the discourse transcription to facilitate the analysis (Du Bois, Cumming, Coburn, and Paolino 1992). Du Bois et al. (1992, 2), 
stated that, discourse convention is "the process of creating a representation in writing of a speech event, in such a way as to make it accessible to discourse research". The researchers decided to use discourse convention because it could provide interesting insights into the nature of the discourse. Moreover, it could enable them to be aware of the special significance of small cues of a linguistic, nonlinguistic or paralinguistic nature.

\subsection{Data Analysis and Coding Validity and Reliability}

The frameworks developed by Hayakawa (2003), and Murata and Hori (2007) were adapted for this research. The original laughter classification framework remained unchanged; however, the names of laughter types were changed for ease of understanding. Thus, to be able to ensure that the adapted version was reliable, the researchers had to initially check the validity of the coding scheme and the reliability of how laughter was coded. The data was coded by two separate coders, a British instructor and a Thai instructor teaching English in leading public universities. This assisted in establishing the inter-coders reliability and reducing socio-cultural bias towards laughter coding.

In the beginning, the researchers provided both inter-coders with the meeting and restaurant transcriptions, audio recordings and the laughter classification scheme along with instructions of how to classify the types of laughter. The following is the coding scheme used in this study.

Classification of Laughter Adapted from Hayakawa (2003), and Murata and Hori (2007)

1. Happy laughter $(\mathrm{HL})$ :

2. Nervous laughter (NL):

3. Laughter of agreement (LA):

4 Threat softening laughter (TSL):

5. Self-deprecating laughter (SDL):
The laughter occurs when the speaker thinks an ongoing conversation is amusing, and the shared laughter is expected.

The laughter is used to soften the atmosphere of serious talk.

The laughter is used to express agreement and create enjoyable conversations.

The laughter happens when the utterance is considered threatening to the listener's feelings or face to soften the tone.

The laughter is used when the speaker feels embarrassed or ashamed about what $\mathrm{s} / \mathrm{he}$ is doing or saying. 
6. In-filling laughter ( $\mathrm{IFL})$ :

7. Purposeless laughter (PL):

8. Laughter of evasion (LE):

9. Backchannel laughter (BCL):
The laughter is used in maintaining the speaker's turn.

The laughter has no clear purposes. It occurs to make the conversation more cooperative.

The laughter happens when the participants don't want to say something clearly or don't want to answer to a question.

The laughter means "I'm listening to you" or "I'm following you."

After receiving the results from the two inter-coders, the points where both disagreed were further discussed and reviewed among the two inter-coders and the researchers. Then the data was reanalyzed to make the results clearer and more reliable.

During the business meeting and brunch at the restaurant, laughter was found throughout the whole of both conversations as demonstrated in the table above (Table 1). Further, it was clearly seen that in the more casual context, the restaurant, laughter occurred more frequently, every o.86 minute; compared to laughter in the meeting every 2.27 minutes. It is possible that the atmosphere in the restaurant was more relaxed than in the meeting which allowed freedom and opportunity for laughter to occur in the conversations.

As mentioned above, the context in which laughter occurred was one of the factors influencing how each laughter type was produced. To illustrate, a meeting is regarded as a more formal and serious discussion and seems to have notable stages to enable the participants to follow and achieve the meeting

TABLE 1 Summary of occurrence of laughter in the meeting and in the restaurant

\begin{tabular}{lccc}
\hline Context & $\begin{array}{c}\text { Period of } \\
\text { recording } \\
\text { (minutes) }\end{array}$ & $\begin{array}{c}\text { Laughter occurring } \\
\text { in a meeting } \\
\text { (times) }\end{array}$ & $\begin{array}{c}\text { Average frequency of } \\
\text { occurrence of laughter } \\
\text { (minutes) }\end{array}$ \\
\hline Restaurant & 32 & 37 & 0.86 \\
Meeting & 134 & 59 & 2.27 \\
\hline
\end{tabular}


goals (Holmes and Stubbe 2003). The transition from one stage to another is quite distinct compared to what happened in the restaurant. Thus, when the participants were in the meeting, they tended to be more prepared and formal since the discussion was mostly about work. Therefore, less laughter was identified in the business meeting context while in the restaurant, with its pleasant ambience, the participants seemed to feel more at ease and it seemed to be a great opportunity for laughter to happen.

Apart from the context, it is noted that a number of the Burmese research participants were not equal in the two contexts. To clarify, in the restaurant, there was only one Burmese participant, while three Burmese participants were present in the meeting. On the other hand, the number of Thai research participants stayed the same in both contexts. Nonetheless, there was no adequate evidence to confirm that the number of participants could be a factor affecting the use of laughter in terms of quantity and types of laughter as can be seen from the results illustrated below.

Figure 1 demonstrates the average percentage of laughter which occurred in the conversations in the restaurant and meeting contexts. It can be seen that in both contexts, the Burmese participants used a wider range of types of laughter compared to the Thai participants (i.e., Burmese participants in the restaurant context -8 types; Thai participants in the restaurant context 3 types; Burmese participants in the meeting context -9 types; Thai participants in the meeting context- 6 types). "In-filling laughter" was the only one type which the Burmese participant did not employ in the restaurant. From

\section{Average Percentage of Laughter Happening in Two Contexts}

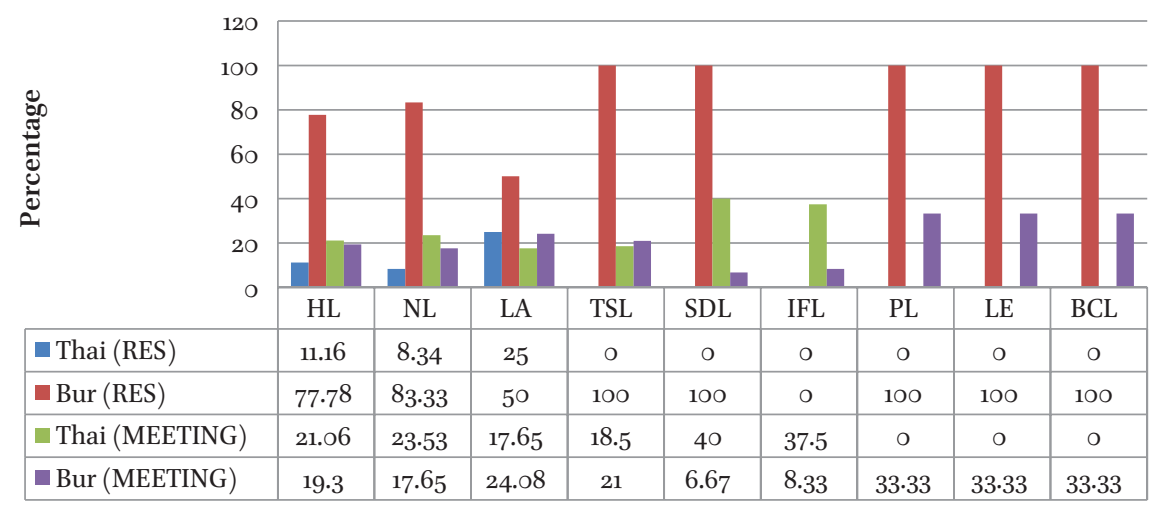

FIGURE 1 Average percentage of laughter happening in the restaurant and in the meeting between Thai and Burmese professionals 
the findings, laughter can probably be assumed to be a common feature of Burmese communication.

It appears that in the restaurant with a Burmese research participant, the average frequency of laughter of all types was notably high (e.g., happy laughter-77.78\%; nervous laughter-83.33\% and threat softening laughter-100\%). On the other hand, in the meeting where three Burmese participants appeared, the average percentage of laughter occurring was relatively lower (e.g., happy laughter-19.3\%; nervous laughter-17.65\% and threat softening laughter-21\%). With regard to Thai participants, even though the number of Thai participants remained the same, the average percentage of laughter between the two contexts was rather different for most types of laughter (e.g., happy laughter in the restaurant-11.16; happy laughter in the meeting-21.06 and nervous laughter in the restaurant-8.34; nervous laughter in the meeting-23.53). Therefore, it is apparent that the number of participants was not an actual factor which had an impact on the quantity of laughter provoked.

In addition, the participants from both countries tended to have similar perceptions of laughter and its interpretation. From the observation, even in a situation where laughter should not occur, for example, in the meeting when they were discussing the issue of the project deadline, laughter was detected and no misinterpretation occurred. Thus, it can be assumed that the perception of laughter use between the research participants from both countries is that it was acceptable behavior. Although, more types of laughter produced by the Burmese participants were recognized compared to the Thai participants, no misconception over the use of laughter was found. Regarding Figure 1, even though the Burmese participant was responsible for a considerable proportion of laughter occurring in the restaurant, no awkward situations or uncomfortable feelings arose.

Other than examining what factors triggered laughter, it is also interesting to explore and compare the percentage of each laughter type which occurred in both contexts to analyze what function laughter could perform. From the findings (Figure 2) depicts various types of laughter which were used for different purposes. Amongst nine types of laughter, happy laughter was the most common one in the meeting and the restaurant settings. However, its propositions (50\% and $27.94 \%)$ are notably different in both settings. In addition to happy laughter, laughter of agreement ( $5.41 \%$ and $16.17 \%)$, threat softening laughter $(2.7 \%$ and $11.76 \%)$, and laughter of evasion (10.81\% and $1.94 \%)$ are also worth discussing as they represent reasonable proportions which are markedly different in the two settings. 


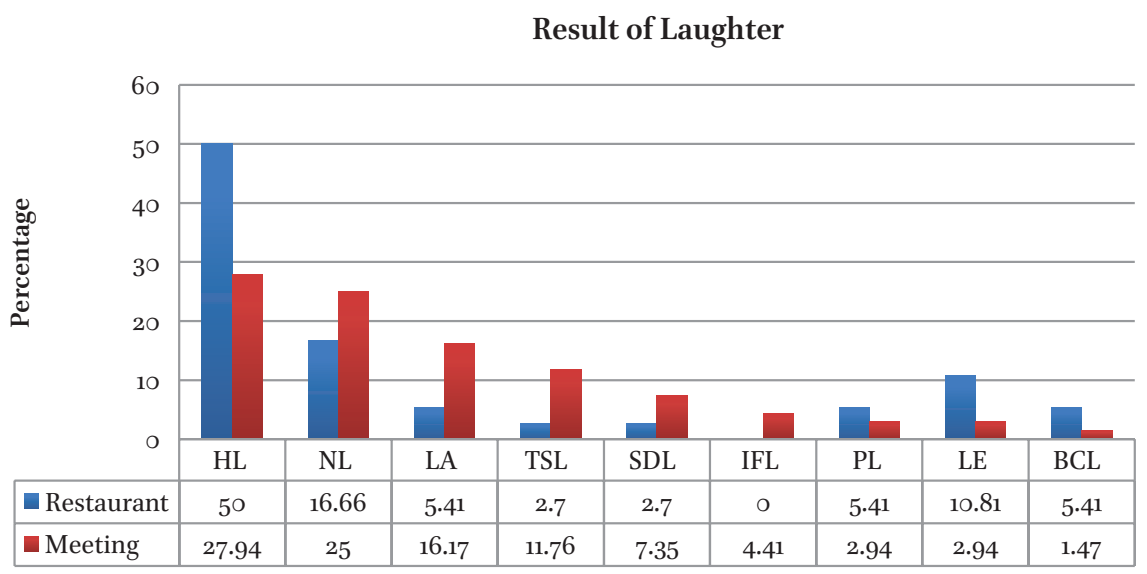

FIGURE 2 Result of laughter between in the meeting and in the restaurant in percentage

Below, four striking types of laughter and the remaining types of laughter discovered in the conversations in two different contexts between Thai and Burmese business professionals are elaborated.

\subsection{Happy Laughter}

Laughter is often perceived as an indicator of joy and happiness which is usually associated with something funny in order to make people laugh. Interestingly enough, happy laughter was ranked first in the context of the restaurant and the business meeting where people might expect discussions to be formal and serious and humor to be less common. From the findings of this study and contrary to general expectations, happy laughter which accounted for 27.94 (meeting) and 50 (restaurant) percent of the happy laughter classification implied certain character traits of this professional group. Hays (2014) noted in his article that; Thais are known for their laid-back lifestyle and attitude towards work; Burmese people, meanwhile, are considered informal, and when they become co-workers, they tend to share similar values and transfer each other's ethics to their business transactions.

Happy laughter can be associated with bonding which has been developed between Thai and Burmese business people since the first day they started working together. The reason why they placed a high value on interpersonal relations was possibly because they were from the same cultural background which is collectivism (Hofstede 1980). In other words, people coming from a collectivist culture lay emphasis on maintaining harmony within interpersonal relationships rather than individual's needs. Hence, it is common practice for Thai and Burmese co-workers to enjoy their time on non-work-related issues such as lifestyle, personal interests and other small talk topics. This type 
of talk is seen as a strategy for managing interpersonal distance and it assists in lubricating social interactions.

Even though happy laughter was the most frequent type of laughter occurring in both contexts, there was a difference in terms of expression of laughter. From the observation and semi-structured interviews with Thai and Burmese participants, it was obvious that when the participants were in the meeting room, they laughed relatively more loudly than when they were in the restaurant. The way they produced laughs at the restaurant sounded noticeably more reserved. In a later interview, Th1 stated that "I could not laugh loudly while we were in the restaurant because we were in public. It was not the same as when we were in the office where we were alone." Mi also pointed out that "when you are in public places, it is better and safer to behave yourself in public in common social situations." This signified that although the relaxing atmosphere at the restaurant affected the frequency of happy laughter to occur, privacy might be another issue which had an influence on how they expressed laughter. Interestingly, the way the participants behaved is in accord with Welty's argument (2016). He mentioned that Thais, especially elders, find great value in being "moh som" (proper) which includes the way one behaves in public. It is plausible that not only Thais consider being "moh som" of great value, but also people in neighboring countries such as Myanmar (Yin 2016, 3).

\subsection{Laughter of Agreement}

Agreement is a daily occurrence in human interactions which range from a conversation at home to a serious business discussion. Agreement is frequently expressed verbally such as "I couldn't agree more" or "Exactly," however, nonverbal behavioral cues along with these expressions or maybe nonverbal behavior alone e.g., smile or laughter could also signify "agreement."

From the findings, in the meeting, laughter of agreement represented $16.17 \%$, while it was $5.41 \%$ in the conversation at the restaurant. This implies that a meeting was a place where the participants discussed, threw in their thoughts and comments about issues and made decisions. Thus, laughter of agreement was found a lot more than in the restaurant.

According to Lewis (2005), the communication style in Asian culture is considered introvert, modest and quiet. Furthermore, Asian countries such as Thailand and Myanmar can be seen as countries in the list of High Context (HC) culture due to the fact that Thai and Burmese communication styles are indirect and rely on contextual cues when conversing (Hall and Hall 1990). Therefore, in certain situations in this study, laughter was selected to assist, support the use of verbal messages for example, "yeah," "I agree," and convey implicitly the meaning of agreement. 
Further, from the findings, it was found that Thai and Burmese participants used verbal messages such as "yeah" and sometimes they had some words overlapping in their conversations together with laughter to indicate "agreement". Undoubtedly, laughter of agreement does not only create a good working atmosphere but it also builds social bonds and shows in-group solidarity. In addition, this phenomenon could somehow provide interesting insight into the supportive relationship between Thai and Burmese business people and the working climate of an organization (Georganta, Panagopoulou, and Montgomery 2014).

\subsection{Threat Softening Laughter}

Threat softening laughter usually occurred when the speaker perceived that an utterance was too aggressive and wanted to use laughter to preserve the face of the listeners whilst still conveying the seriousness of intention in a friendly way. Hays (2014) suggested that Southeast Asian people living in a nonconfrontational society are polite. Thus, a public dispute or criticism is usually avoided. In addition, Claramita, Nugraheni, and van Dalen (2013, 24) affirmed that "Southeast Asian culture places high value on harmonious relations and to avoid conflict" which accords with Kardkarnklai's study (2009) that Asian people tend to avoid conflicts. Nonetheless, in some cases, when they had to confront the conflict, they would rather handle it their own way. In this study, laughter, needless to say, was selected as a tool to soften any threat between Thai and Burmese business people.

According to the findings, it was obvious that threat softening laughter occurred more frequently in the meeting (11.76\%) than in the restaurant $(2.7 \%)$. This was because during the meal, they did not talk much about work and even when they discussed work issues; it was in a more relaxed way. Further, work-related issues being discussed in the restaurant were less serious and the information exchanged between two parties was not in depth compared to what happened in the office meeting. Nonetheless, there was a time when threat softening laughter was found while they were having brunch. It occurred when they were talking about an ongoing project, specifically time constraints and project deadlines. Before the threat softening laughter happened, Th1 and Mi agreed on the point that they needed to finish this project as soon as possible. However, one of the main problems that Mi worried about was planning; therefore, he asked Thı how Thı had outlined an action plan to finish this project. M1 then continued his talk implying that the owner put him under pressure and he wanted to let Thi know he had to take serious action. However, as Southeast Asian people favor compromising to avoid conflicts; M1 used a laughing tone in his voice when he mentioned that the main contractor's work 
had gone very fast in order to deliver an implied threat to Thı that Thı had to catch up with the schedule and complete the project. The laughing tone in M1 voice was meant to soften the threat he made and not sound "too aggressive." Thus, M1 decided to apply this laughter strategy to make the statement benign but firm. Meanwhile Thi realized the sense of seriousness and understood the implied message that M1 conveyed as he agreed that he needed to revise the plan to keep up the project work.

\subsection{Laughter of Evasion}

There are a number of ways to politely avoid answering a question and keep the ongoing conversation friendly and avoid sounding disrespectful such as re-questioning the questioner or changing the subject. In everyday or business meeting conversations, it is possible that we have to confront questions which we do not wish to reply to or sometimes we cannot come up with appropriate answers. Instead of the solutions mentioned earlier, laughter could be another option.

According to the transcribed data, laughter of evasion happened when the participants deliberately avoided talking about something or answering a question from another interlocutor. From the findings, evasion laughter occurred five times more frequently in the restaurant (10.81\%) than in the meeting $(2.94 \%)$. This was because the meeting was a place where the participants brainstorm ideas (Reinig and Shin 2003; Volkema and Niederman 1995) and make comments. Thus, it was difficult to avoid answering questions. This was also in accord with interviews with the research participants. Th1 stated that it was important that questions needed to be answered as the ideas, comments, and information gained from the meeting were crucial in decision making. Meanwhile, Mi provided an interesting thought that before a meeting, everyone should be prepared; otherwise, it would be a waste of time. Since Thai participants had to go to Yangon to have face-to-face meetings, both Thai and Burmese participants wanted to spend their time wisely.

However, in the restaurant, laughter of evasion was $10.81 \%$ due to the relaxing atmosphere and the topics of the talk. Both $\mathrm{M} 1$ and Th1 mentioned that during brunch, they were in pleasant surroundings; they were, therefore, relaxed. Noticeably, laughter of evasion happened mostly when the participants were talking about non-work-related issues or small talk for example, English vocabulary, the Burmese boss's health, and Thı's iPhone. M1 remarked that in the restaurant, unlike in the meeting, it was acceptable not to give clear answers, it rarely happened and he continued that laughter was used to help maintain a positive and optimistic outlook through these situations. 


\subsection{Other Types of Laughter}

In addition to the most markedly different types of laughter between Thai and Burmese participants, five other forms of laughter occurred and are explained in the following.

\subsubsection{Nervous Laughter}

With regard to nervous laughter, it aims to soften the atmosphere of seriousness of the talk for example, in conflict or negotiating situations. Thus, from the findings (Figure 2), in both contexts where negotiated situations happened, nervous laughter occurred in order to handle the situations (in the restaurant-16.66\%; in the meeting-25\%).

However, from the findings, the interplay of nervous laughter as a communication strategy between Thai and Burmese business persons was rather different. Even though both Thai and Burmese participants employed nervous laughter as a strategy, the proportion of this laughter in the two contexts was not the same. From the results (Figure 1), for Thai's usage, nervous laughter was identified $8.34 \%$ in the restaurant and $23.53 \%$ in the meeting. On the other hand, for Burmese usage, it was recognized $83.33 \%$ in the restaurant and $17.65 \%$ in the meeting. Noticeably, the Thai participants used this particular type of laughter only when it was a work-related topic (e.g. salary problems and coordination work), while the Burmese participants preferred to use nervous laughter when the topics were about non-work (e.g. menu and gossip). This could be because in a more casual context, the Thai participants did not pay much attention to the conflicts happening or they might not even realize that there were disagreements arising. In contrast to the Thai participants, the Burmese participants were hosts and therefore, it was their responsibility to take good care of their "visitors" which included making the atmosphere of the talks and the feelings of interlocutors better when they became tense.

\subsubsection{Self-Deprecating Laughter}

Noticeably, during the discussion, there were times when the research participants were joking around and from the research data, it can be seen that they sometimes employed self-deprecating humor which means they laughed at their imperfections or shortcomings and most of the time, this type of laughter could elicit shared laughter. To elucidate, in the transcribed data, the use of self-deprecating laughter was found in both contexts and especially, in the meeting it happened at the beginning, in the middle and at the end of the talk. It is of interest to note that the proportion of self-deprecating laughter between Thai and Burmese participants in both contexts was distinct (Figure 1). Regarding the Thai participants, they employed this strategy only in the 
meeting because they realized that there should be a break from serious conversations. However, playing a joke on co-workers might not be considered appropriate especially in the meeting situation. Thus, it seemed to be "safer" to make funny remarks about themselves. On the other hand, the Burmese participants tended to view self-deprecating laughter as a good strategy to apply in both serious and casual contexts.

According to Figure 2, although the proportion of this laughter type was not high (in the meeting $-7.35 \%$; in the restaurant $-2.7 \%$ ), it seemed to be a useful communication strategy for both Thai and Burmese participants and it was considered successful because when this laughter type was employed, they shared a laugh. It is believed that certain types of humor in the workplace can foster a positive atmosphere that helps co-workers bond (Hoption, Barling, and Turner 2013). In addition, self-deprecating humor and self-deprecating laughter function as a stimulus to build trust among them and it usually breaks down the wall between co-workers. Maintaining a healthy working atmosphere by laughing at themselves but not their colleagues, may be seen as more likeable and caring.

\subsubsection{In-Filling Laughter}

Other than using verbal communication such as "let me continue" implying to other interlocutors that the speech is not finished or using non-verbal communication such as putting your hand up, laughter could be an interesting option for the speaker to maintain his/her turn.

The type of laughter which was identified $4.41 \%$ and discovered only in the meeting was in-filling laughter. From the findings (Figure 1), the proportion of this laughter type of the Thai participants $(37.5 \%)$ was very high compared to the Burmese participants (8.33\%). It is because in the meeting, Th1 acted as a meeting leader, thus most of the time he was the one who spoke. This type of laughter could assist him in holding the floor until he had finished. While in the restaurant conversation, there was none to be found (Figure 2). It was not because the act of interrupting did not occur but it was because both Thai and Burmese participants either ignored each other's interruptions and continued their talk or simply allowed the interruptions to occur. It can be concluded that in-filling laughter might not be a necessary communication strategy in an informal context such as a restaurant.

\subsubsection{Purposeless Laughter}

Types of laughter, as stated previously, served different roles and functions. As for purposeless laughter, the research participants were likely to use it as a communication strategy only to have a smooth conversation. An interesting 
point observed from the data is that purposeless laughter usually followed a statement or a question which was not humorous and the reason for the laughter could not be detected. From the research data, this type of laughter was also found from time to time in work-related discussion and small talk in both contexts (Figure 2 : the restaurant $-5.41 \%$; the meeting $-2.94 \%$ ). Additionally, from Figure 2, it can be observed that purposeless laughter seemed to be a preferred strategy employed by the Burmese participants as they used it both in the restaurant $(100 \%)$ and in the meeting $(33.33 \%)$, whilst the Thai participants never used this strategy. Also, when this strategy was used, laughter was not shared by all the members of the group as it is possible that the Thai participants did not understand why the Burmese participants laughed and they decided to ignore it.

\subsubsection{Back Channeling Laughter}

According to Dwyer $(2006,14)$, it is necessary for "both sender and receiver to have interactive skills" to achieve successful communication. There are different types and levels of listening skills, for example, attending listening, encouraging listening, reflecting listening and active listening. In terms of back channeling laughter, it is categorized into active listening because this type of laughter acts as a response to the speaker which means "I'm following you or I hear you."

From Figure 2, although the proportion of this type of laughter was small compared to other laughter types, it still occurred in both contexts (in the restaurant -5.41 ; in the meeting 1.47). More interestingly, back channeling laughter was employed only by the Burmese participants (in the restaurant - 100\%; in the meeting - 33.33\%). It can be noted that it was their preferred communication strategy to show their attentive listening. While, for Thai participants, they applied other techniques such as smiling and nodding to show that they were listening.

\section{5}

\section{Conclusion}

Understanding cultural and behavioral perspectives of international business people from different countries is becoming more important in our increasingly interconnected global economy. This study demonstrates that laughter is a meaningful element in business conversations. Even if it might be produced unintentionally, it usually conveys certain contextual meanings. Furthermore, it should be noted that laughter can be interpreted differently by individuals from different cultures. The participants in this study, fortunately, were from 
two close neighboring countries and seem to share a similar cultural background to some extent as they did not have problems interpreting the use of laughter of one another in different situations. In addition, their use and perception of the laughter did not hinder successful business communication at all. Instead, laughter helped the flow of communication. To them, laughter is considered a "communication key" to lead the way to successful and understandable communication.

From the results, it can be observed that different types of laughter were used in formal and informal contexts with different purposes. This confirms that laughter can happen in a specific context, for example jury deliberations during a capital murder case (Keyton and Beck 2010) and business discourse (Hayakawa 2003) since laughter is used as a communication tool strategically and intentionally. Happy laughter, which occurred $27.94 \%$ in the meeting and $50 \%$ in the restaurant, was the most distinctive in both settings amongst nine types of laughter. This signifies the uniqueness of characteristic traits of Thai and Burmese business people such as their laid-back personality and their values in maintaining good business relationships. According to Kardkarnklai's study (2009), positive talk in an amusing way not only assists in keeping a close relationship but also promotes a relaxing atmosphere and softens possible conflicts happening during discussion. Intriguingly, it was found there were no misinterpretations or fallacies found in this study; both Thai and Burmese business people knew when it was appropriate to produce and use laughter and when laughter was needed in their discussion. In brief, the study reveals that when laughter is produced, it does not only imply certain meanings to other interlocutors, it also connotes the cultural aspects to the group members who are from different countries. The results of this research demonstrate interesting and thought-provoking messages about the use of laughter in these particular contexts. In addition, they shared and understood their workplace communication discourse and to a certain extent each other's culture.

\section{References}

Andrews, Deborah. 2016. "A Space for Place in Business Communication Research." International Journal of Business Communication 54(3) (November): 325-336. https://doi.org/ 10.1177/2329488416675842.

Bachorowski Jo-Anne, and Michael Owren. 20o1. "Not all Laughs are Alike: Voiced but not Unvoiced Laughter Readily Elicits Positive Affect." Psychological Science 12(3) (May): 252-257. https://doi.org/ 10.1111/1467-9280.00346. 
Bonin, Francesca. Nick Campbell, and Carl Vogel. 2014. "Time for Laughter." KnowledgeBased Systems 71 (November): 15-24. https://doi.org/10.1016/j.knosys.2014.04.031.

Brain, Marshall. 200o. "How Laughter Works." Howstuffworks. Accessed on December 12, 2019. https://science.howstuffworks.com/life/inside-themind/emotions/ laughter.htm.

Brock, Alexander. 2016. “The Borders of Humorous Intent - The Case of Tv Comedies.” Journal of Pragmatics 95 (April): 58-66. https://doi.org/10.1016/j.pragma.2015.12.

Claramita, Mora, Mubarika Nugraheni, and Jan van Dalen. 2013. "Doctor-patient Communication in Southeast Asia: A Different Culture?." Advances in Health Sciences Education: Theory and Practice 18(1) (March):15-31. https://doi.org/10.1007/ s10459-012-9352-5.

Clift, Rebecca. 2016. "Don't Make Me Laugh: Responsive Laughter in (Dis)affiliation.” Journal of Pragmatics 100 (July): 73-88. https://doi.org/10.1016/j.pragma.2016.01.012.

Crooke, Nancy, Jamie Gorman, Christopher Myers, and Jasmine Duran. 2013. "Interactive Team Cognition." Cognitive Science 37(2) (March): 255-285. https://doi. org/10.1111/cogs.120o9.

Du Bois, John, Susanna Cumming, Stephan Schuetze Coburn, and Danae Paolino. 1992. "Discourse Transcription." Santa Barbara Papers in Linguistics 4: 1-225.

Dwyer, Judith. 20o6. The Business Communication Handbook, 7 th ed. Australia: Pearson.

Forgas, Joseph Paul. 1985. Interpersonal Behaviour: The Psychology of Social Interaction. Oxford: Pergamon Press.

Georganta, Katerina, Efharis Panagopoulou, and Anthony Montgomery. 2014. "Talking behind their Backs: Negative Gossip and Burnout in Hospitals." Burnout Research 1(2) (September): 76-81. https://doi.org/10.1016/j.burn.2014.07.003.

Gervais, Matthew, and David Wilson. 2005. "The Evolution and Functions of Laughter and Humor: A Synthetic Approach." Quarterly Review of Biology 80(4) (December): 395-43o. https://doi.org/10.1086/498281.

Ginzburg, Jonathan, Ellen Breitholtz, Robin Cooper, Julian Hough, and Ye Tian. 2015. Understanding Laughter. Paper presented at the Proceedings of the 2oth Amsterdam Colloquium, 16-18 December, 2015, Netherlands.

Glenn, Phillip. 2010. "Interviewer Laughs: Shared Laughter and Asymmetries in Employment Interviews." Journal of Pragmatics 42(6) (June): 1485-1498. https://doi. org/10.1016/j.pragma.2010.01.oog.

Godkewitsch, Michael. 1976. "Physiological and Verbal Indices of Arousal in Rated Humour." In Humour and Laughter: Theory Research and Applications, edited by Anthony Chapman, Hugh Foot, and Peter Derks, 117-138. London: Wiley.

Hall, Edward, and Mildred Hall. 1990. Understanding Cultural Differences: Germans, French and Americans. Yarmouth: Intercultural Press. 
Handford, Michael, and Matous, Petr. 2015. "Problem-Solving Discourse on an International Construction Site: Patterns and Practices." English for Specific Purposes 38 (April): 85-98. https://doi.org/ 10.1016/j.esp.2014.12.002.

Hayakawa, Haruko. 2003. "The Meaningless Laugh: Laughter in Japanese Communication." Ph.D. Diss., University of Sydney.

Hays, Jeff. 2014. "Facts and Details." Facts and Details: Southeast Asia, December 7, 2018. http://factsanddetails.com/southeast-asia.

Hofstede, Geerl. 1980. Culture's Consequences: International Differences in Work-related Values. California: Sage Publications.

Holmes, Janet, and Maria Stubbe. 2003. Power and Politeness in the Workplace. London: Longman.

Hoption, Colette, Julian Barling, and Nick Turner. 2013. "It's Not You, it's Me: Transformational Leadership and Self-deprecating Humor." Leadership \& Organization Development Journal 34(1) (February): 4-19. https://doi.org/ 10.1108/ o1437731311289947.

Kardkarnklai, U-maporn. 20og. "Conflict-softening in Thai-Japanese Business Discourse." In New approaches to Discourse and Business Communication, edited by Ramallo Fernado. Anxo M. Lorenzo, Xoán Paulo Rodriguez-Yáñez, and Piotr Cap, 153-170. London: Palgrave Macmillan.

Keyton, Joann, and Stephenson J. Beck. 2010. "Examining Laughter Functionality in Jury Deliberations." Small Group Research 41(4) (July): 386-407. https://doi. org/10.1177/1046496410366311.

Lehmann-Willenbrock, Nale, and Joseph Allen. 2014. "How Fun are your Meetings? Investigating the Relationship between Humor Patterns in Team Interactions and Team Performance." Journal of Applied Psychology 99(6) (November): 1278-1287. https://doi.org/10.1037/aoo38083.

Lewis, Richard Donald. 2005. Finland, Cultural Lone Wolf. Yarmouth: Intercultural Press.

Lyttle, Jim. 2007. "The Judicious Use and Management of Humor in the Workplace." Business Horizons 5o(3) (May-June): 239-245. https://doi.org/10.1016/j.bushor. 2006.11.001.

Marakaki, Vassiliki, Sara Merlino, Lorenzo Mondada, and Florence Oloff. 2010. "Laughter in Professional Meetings: The Organization of an Emergent Ethnic Joke." Journal of Pragmatics 42(6) (January): 1526-1542. https://doi.org/10.1016/j. pragma.2010.01.013 V.

Meyer, John. 2000. "Humor as a Double-Edged Sword: Four Functions of Humor in Communication." Communication Theory 10(3) (March): 310-331. https://doi. org/10.1111/j.1468-2885.20oo.tboo194.x. 
Murata, Kazuyo. 2014. "An Empirical Cross-cultural Study of Humour in Business Meetings in New Zealand and Japan." Journal of Pragmatics 6o (January): 251-265. https://doi.org/10.1016/j.pragma.2013.o9.002.

Murata, Kazuyo, and Motoko Hori. 2007. "Functions of Laughter in Intercultural Communication: An Analysis of Laughter as a Communicative Strategy." Kokusai Shakai Bunka Kenkyujo Kyou 9: 115-124.

Ojha Kumar Ajay, and Tammy Holmes. 2010. "Don't tease me, I'm working: Examining Humor in a Midwestern Organization using Ethnography of Communication." The Qualitative Report 15(2) (March): 279-30o. https://doi.org/ 10.46743/216o-3715/2010.1152.

Oveis, Christopher, Aleksandr Spectre, Pamela Smith, Mary Liu, and Dacher Keltner. 2016. "Laughter Conveys Status." Journal of Experimental Social Psychology 65 (April): 109-115. https://doi.org/10.1016/J.JESP.2016.04.005.

Provine, Robert. 2013. "Laughing, Grooming, and Pub Science." Trends in Cognitive Sciences 17(1) (January): 9-10. https://doi.org/10.1016/j.tics.2012.11.oo1.

Reinig, Bruce, and Bongsik Shin. 2003. "The Dynamic Effects of Group Support Systems on Group Meetings." Journal of Management Information Systems 19(2) (Fall): $303-325$.

Ritter, Jan, Carolin Brück, Heike Jacob, Dirk Wildgruber, and Benjamin Kreifelts. 2015. "Laughter Perception in Social Anxiety." Journal of Psychiatric Research 60 (September): 178-184. https://doi.org/10.1016/j.jpsychires.2014.09.021.

Rogerson-Revell, Pamela. 2007. "Using English for International Business." English for Specific Purposes 26(1) (December): 103-120. https://doi.org/10.1016/j.esp.2005. 12.004 .

Ruch, Willibald, and Paul Ekman. 2001. "The Expressive Pattern of Laughter." In Emotion, Qualia and Consciousness, edited by Alfred Kaszniak, 426-443. Tokyo: Word Scientific Publisher.

Szameitat, Dian, Kai Alter, André Szameitat, Chris Darwin, Dirk Wildgruber, Susanne Dietrich, and Annette Sterr. 2009. "Differentiation of Emotions in Laughter at the Behavioral Level." Emotion 9(3) (June): 397-405. https://doi.org/10.1037/aoo15692. Vöge, Monika. 2008. "All You Need is Laugh: Interactional Implications of Laughter in Business Meetings.” Ph.D. diss., University of Southern Denmark.

Volkema, Roger, and Fred Niederman. 1995. Organizational Meetings: Formats and Information Requirements. Small Group Research 26(1) (February): 3-24. https:// doi.org/10.1177/1046496495261001.

Welty, Roger. 2016. Thai Culture and Society. Bangkok: Thailand Asia Books.

Yin, Saw Myat. 2016. CultureShock! Myanmar: A Survival Guide to Customs and Etiquette. Singapore: Marshall Cavendish Editions. 